\title{
The mystery of "missing" visits in an emergency cardiology department, in the era of COVID-19.; a time-series analysis in a tertiary Greek General Hospital
}

\author{
Konstantinos Tsioufis ${ }^{1}$ (D) . Christina Chrysohoou ${ }^{1} \cdot$ Maria Kariori $^{1} \cdot$ loannis Leontsinis ${ }^{1} \cdot$ Ioannis Dalakouras $^{1}$. \\ Angelos Papanikolaou ${ }^{1} \cdot$ Georgios Charalambus ${ }^{2} \cdot$ Helen Sambatakou $^{3} \cdot$ Gerasimos Siasos $^{1}$. \\ Demosthenes Panagiotakos ${ }^{4} \cdot$ Dimitrios Tousoulis $^{1}$
}

Received: 4 May 2020 / Accepted: 28 May 2020 / Published online: 6 June 2020

(c) Springer-Verlag GmbH Germany, part of Springer Nature 2020, corrected publication 2020

\begin{abstract}
Background In the era of the current COVID-19 health crisis, the aim of the present study was to explore population behavior as regards the visits in the Emergency Cardiology department (ECD) of a tertiary General Hospital that does not hospitalize SARS-CoV-2 infected patients

Methods and results Daily number of visits at the EDC and admissions to Cardiology Wards and Intensive Care Unit of a tertiary General Hospital, in Athens, Greece, were retrieved from hospital's database (January 1st-April 30th 2018, 2019 and 2020). A highly significant reduction in the visits at ECD of the hospital during March and April 2020 was observed as compared with January and February of the same year ( $p$ for linear trend $<\cdot 001$ ); in particular the number of visits was $41.1 \%$ lower in March 2020 and 32.7\% lower in April 2020, as compared to January 2020. As the number of confirmed COVID19 cases throughout the country increased (i.e., from February 26th to April 2nd) the number of visits at ECD decreased $(p=0.01)$, whereas, the opposite was observed in the period afterwards $(p=0.01)$.The number of acute Myocardial infarctions (MI) cases in March 2020 was the lowest compared to the entire three year period ( $p<0 \cdot 001)$; however, the number of acute MI cases in April 2020 was doubled as compared to March 2020, but still was lower than the preceding years $(p<0 \cdot 001)$. Conclusions It is hard to explain the mystery of the "missing" emergency hospital visits. However, if this decline in cardiovascular disease related hospital visits is "true", it is something that needs to be rigorously studied, to learn how to keep these rates down.
\end{abstract}

Keywords Covid $19 \cdot$ Emergency cardiological department $\cdot$ Missing visits

\section{Introduction}

Konstantinos Tsioufis

ktsioufis@hippocratio.gr

1 First Cardiology Clinic, Medical School, National and Kapodistrian University of Athens, Hippokration Hospital, 114 Vass Sofias Ave, 11527 Athens, Greece

2 Emergency Department, Hippocratio Hospital, Athens, Greece

3 2nd Department of Internal Medicine, HIV Unit, Medical School, Hippokration General Hospital, National and Kapodistrian University of Athens, Athens, Greece

4 School of Health Science and Education, Harokopio University, Athens, Greece
The recent Coronavirus Disease (COVID-19) epidemic, caused by Severe Acute Respiratory Syndrome Coronavirus 2 (SARS-COV-2), has resulted in an increased death toll, globally, that has surpassed the combined death toll of previous SARS cases [1, 2]. In addition, the widespread transmission of COVID-19 led the World Heart Organization (WHO) on 11th of March 2020 to declare it as pandemic. Due to the high infectiousness of SARS-COV-2 and the significant morbidity and mortality rates among the affected population, many countries proceeded to preventive measures imposing home-isolation to citizens, limiting and monitoring transportation to the essentials. Regarding the healthcare system and in order to focus on the management of COVID-19 incoming patients, all 
elective procedures in hospitals were postponed, while, only the urgent ones were permitted. However, the recommendations to avoid hospital visits, together with the fear of getting infected by COVID-19, may have forced individuals to underestimate the symptoms of a disease onset, and, therefore, to delay the diagnosis of various, especially acute health conditions, like cardiovascular disease (CVD). Especially in older individuals with various co-morbidities, in which symptoms of acute coronary events are usually less specific, a delay in diagnosis and lack of the appropriate treatment, can lead to rapid deterioration, shock and death [3].

Thus, and in the era of the current COVID-19 health crisis, the aim of the present study was to explore population behavior as regards the visits in the emergency, outpatient cardiology department of a tertiary General Hospital, that does not hospitalize SARS-CoV-2 infected patients. At this point it should be mentioned that the first confirmed COVID-19 case in Greece was reported on February 26th and there was a progressive increasing transmission trend until the April 2nd (published reports at www.eody.gov.gr), while the aforementioned restrictive measures were imposed on the $10^{\text {th }}$ of March.

\section{Methods}

\section{Design}

Observational, retrospective study of the unplanned visits to an Emergency Cardiology Department (ECD) in a tertiary heart center in Greece.

\section{Setting}

The data of daily visits to the outpatient Emergency Cardiology Department (ECD) and the admissions to the two Cardiology Wards and the Cardiology Intensive Care Unit (ICU) of Hippokratio Hospital, a tertiary General Hospital, in Athens metropolitan area, Greece, were retrieved from hospital's database. The hospital covers more than one third of ATTICA region and nearby rural areas. The aggregated data were for the period January 1st-April 30th 2020 and the previous two years, i.e., 2018 and 2019.

\section{Bioethics}

The study was carried out following standard ethics requirements with the permission of the hospital's Bioethics Committee.

\section{Measurements}

From the hospital's database, all unplanned visits in the ECD of the aforementioned period were extracted by sex, age, presenting complaint, initial diagnosis and course (i.e., admission in the cardiology ward, referral to other specialty or discharge). For the current study, the presenting complaints of interest were: chest pain (ICD-10: R07.1, R07.2, R07.8, R07.9); dyspnea (ICD-10:R06.0); elevated blood pressure (ICD-10:I16) and palpitations (ICD10: R0.002;I48).

Furthermore, the diagnosis of the admitted patients was also retrieved through the hospital's database. The principle discharge diagnoses of interest were: acute coronary syndrome [ST-elevation Myocardial Infarction (STEMI) (ICD-10 I22, I21.9, I21, I21.4), non-ST-elevation Myocardial Infarction (Non-STEMI) (ICD-10 I22, I21.4), Unstable angina (ICD-10 I20.0)], heart failure (ICD-10: I50, I42, J81), other manifestations of coronary artery disease (CAD) (ICD 10: I43, E78, I130.8, E78.4, I42.2, I27.2, I71.2, I42.1, I30.9, I71, I10, I33, I31.3, I26, I11, I34, I35.8, I07.1, Q25.3, I35I70, I25.1, I25.8, I25.6, I20.9, I24.5, I24.9, I54, I25)and in-hospital cardiovascular death (ICD-10 I46, I46.9).

\section{Statistical analysis}

Visits are presented as counts. Outpatient visits were analyzed by applying Generalized Estimating Equations with log linear Poisson link function. Additional explanatory variables were day-of-week (six "dummy" variables), holidays (binary variable indicating the dates that are official holidays) and Monday effect (binary variable indicating Monday as a day with a potentially larger number of hospital admissions). Goodnessof-fit of the statistical models was assessed by calculating the dispersion, whose optimum value is 1.0 for the Poisson regression, as well as from the q-q plot of the standardized deviance residuals. Linear-trend model was estimated to evaluate differences in the count of visits between January and April of the three studied years separately (2018, 2019 and 2020). Moreover, non-linear trends (i.e., parabolic and fractional polynomial) were also tested. Cross-correlation analysis was also applied between the time-series of COVID-19 confirmed cases and visits at ECD from February 26th (the first confirmed case in Greece) to April 30th. All tested hypotheses were two-sided. All statistical analyses were carried out using the Stata SE 15 software (STATA Corp Ltd., Texas, USA). 


\section{Results}

\section{ECD visits}

A highly significant reduction in the visits at ECD of the hospital during March and April 2020 was observed, as compared with January and February ( $p$ for linear trend $<.001)$; in particular the number of visits was $41.1 \%$ lower in March 2020 and 32.7\% lower in April 2020, as compared to January 2020. Moreover, the number of visits in March and April 2020 were the lowest compared to the same months, in previous years. Moreover, no significant trends were observed regarding the number of visits during the same period, i.e., January to April, in 2018 and in 2019 (Fig. 1). Cross-correlation analysis showed that as the number of confirmed COVID-19 cases in Greece increased (i.e., from February 26th to April 2nd) the number of visits at ECD decreased $(r=-0.21$, lag 4 days, $p=0.01$ ), whereas, as the number of COVID-19 cases in Greece started to decline (i.e., from April 3rd) the number of visits at ECD increased $(r=+0.18$, lag 9 days, $p=0.01$ ).

No differences were observed during 2018-2020 regarding mean age (mean (SD): 63.5(1.0) years, $p=0.52$ ) and sex of the individuals that visited ECD (62.0\% men, $p=0 \cdot 28$ ). Moreover, no differences were observed in the pattern of presenting complaints (by the individuals who visited the ECD), as well as the percentage of individuals who died at the ECD $(1 \%$, range $0-2 \%$, pfor trend $=0 \cdot 38)$, during the entire three year period 2018-2020 (Fig. 2). In addition, although no differences were observed in the percentage of primary percutaneous coronary intervention (pPCI) performed in the individuals who visited the ECD during the period of 2018 and 2019 and the first two months of $2020(3 \%$, range $2-5 \%, p$ for trend $=0.72)$, a significant reduction by $50 \%$ was observed in March of 2020 compared to January and February $2020(p<0.01)$, which was not evident in April 2020.

\section{Admissions at Cardiology Wards and ICU from the ECD}

Concerning the rate of patients admitted to the Cardiology ward from the ECD, this was on average $42 \%$ throughout the studied period 2018-2020 (range from 38 to 47\%). A significant progressive increase in the admission rate was observed from February to April 2020, as compared to the preceding years; specifically, $44 \%$ of visitors were admitted to the Cardiology ward in February, 45\% in March and 48\% in April ( $p$ for trend $=0.05$ ).Moreover, the rate of patients admitted to the Cardiology ward in March and April 2020 was significantly higher compared to the previous years (2018 and 2019), ( $p=0.006$ and $p<0 \cdot 001$, respectively). No differences were observed for the entire three-year period as regards the mean age (mean (SD) 68.1(1.0) years, $p=0.07$ ) and the sex (66.9\% men, $p=0.53)$ of the admitted individuals. The pattern of the principle discharge diagnoses of interest [Acute coronary syndrome (ACS), Heart failure and other manifestations of CAD (including chronic atherosclerotic disease,
Fig. 1 Monthly unscheduled visits at the ECD of a tertiary General Hospital, during January 2018-April 2020 ( $p$-values derived from linear trend analysis, lines derived from fractional polynomials per year of study); time-series line (bottom right) represent the confirmed COVID-19 cases in Greece, from February 26th to April 30th, 2020 (note: the peak observed on April 21st is due to a series of cases observed in a refugee/immigrants campus)

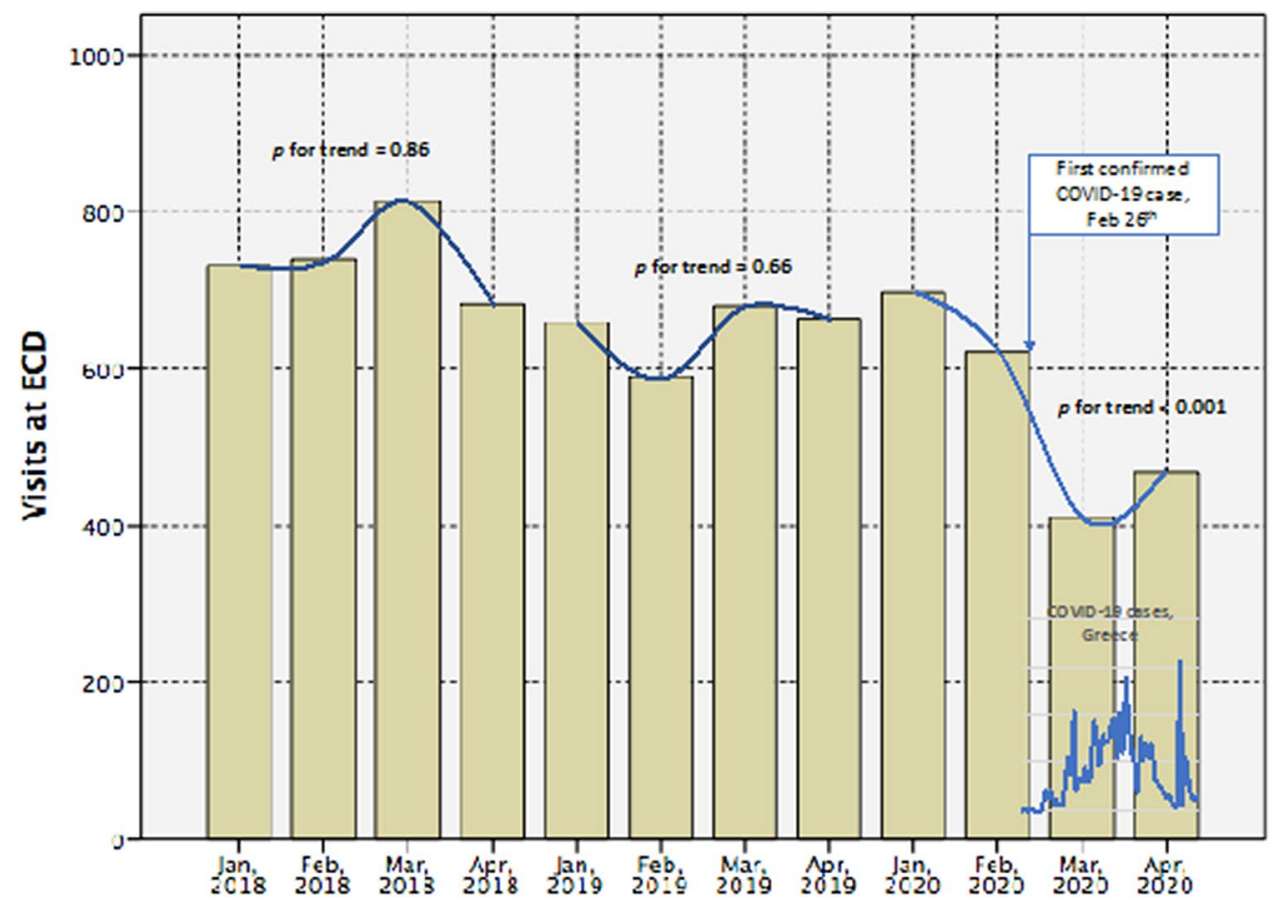


Fig. 2 Pattern of the presenting complaints among unplanned visits in the ECD of a tertiary General Hospital, in Athens, Greece, during January 2018April 2020

Fig. 3 Pattern of major discharge diagnoses: acute coronary syndromes (ACS), heart failure (HF) and other coronary artery disease (CAD) which includes chronic atherosclerotic disease, stable angina and semielective percutaneous procedures of the patients who admitted to the Cardiology Wards of a tertiary General Hospital, in Athens, Greece, during January 2018-April 2020
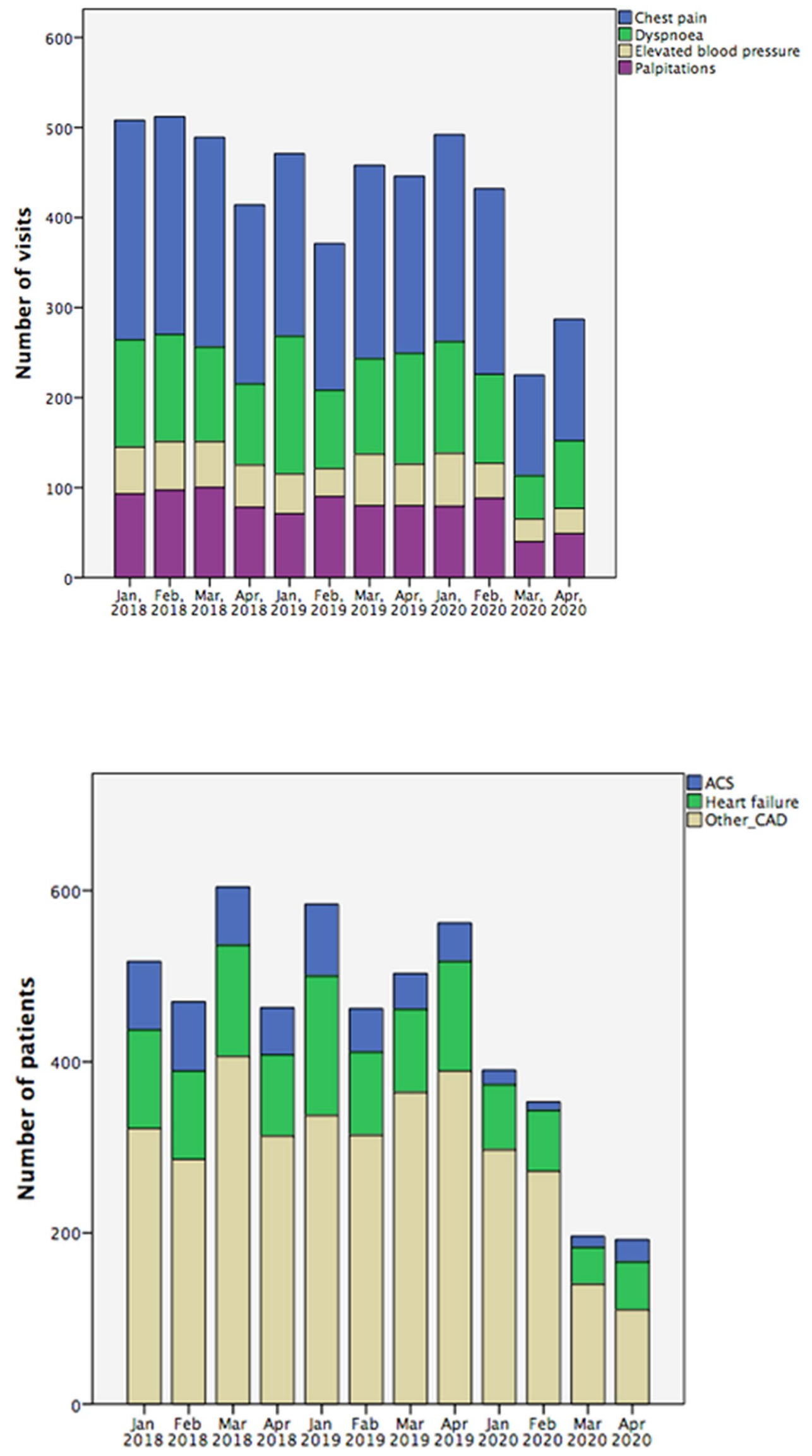
stable angina and semi-elective percutaneous procedures)] for the patients admitted to the cardiology ward is presented in Fig. 3. The number of acute myocardial infarction cases in March 2020 was the lowest compared to the entire three year period ( $n=13$ cases vs. 95\% CI 35-65, $p<0 \cdot 001$ ); however, the number of acute myocardial infraction cases in April 2020 doubled as compared to March 2020, but still it was lower than the preceding years $(n=26$ cases, $p<0 \cdot 001)$. Similar significant findings were observed when STEMI and Non-STEMI cases were studied separately. Regarding heart failure, the number of admitted patients in March 2020 was the lowest compared to the entire period ( $n=43$ cases vs. 95\%CI 53-92, $p<0 \cdot 001$ ), whereas the number admitted in April was the second lower ever observed $(n=56$ cases, $p<0 \cdot 001$ ). Finally, regarding other CAD cases (including semi-elective procedures) the number observed in March $(n=140)$ and April $2020(n=110)$ were respectively 3.1and 2.4-times lower compared to the preceding period (vs. $95 \% 167-236, p<0 \cdot 001)$.

\section{Discussion}

The present study reports a strong reduction in all cause visits at the ECD of a tertiary General Hospital during the COVID-19 outbreak in Athens, Greece. The reduction was more evident in the period that COVID-19 cases increased and less profound when the epidemic curve tended to be flattened. Greek government imposed general lockdown measures on the 10th of March. Our center, implemented the restrictions regarding elective cases, so no elective coronary case was performed. This political action included school closure. Moreover, major public holidays and religious events were transferred to a later date. This possibly explains the fact that we detected no impact of school operation or public holidays on our analysis. The public was in general encouraged to stay at home and seek for medical care only in case of alerting symptoms. This policy has probably had a significant contribution to the aforementioned reported reduction in all-cause visits to the ECD.

It is well established a link between viral infections and an increased incidence of acute cardiovascular events, mainly due to the impact of inflammatory process leading to atherosclerotic plaque rupture. However, in the era of SARSCov-2 outbreak, a significant reduction by $50 \%$ was observed in March of 2020 compared to February 2020 in the percentage of primary PCI performed in individuals who visited the ECD. This reduction was somewhat restored in April 2020 (especially the last 10 days of April).In agreement with our findings, a Spanish study that recorded visits from 71 hospitals of the STEMI care network in Spain reported a $40 \%$ reduction in patients treated with primary PCI [4]. In another study conducted in 9 high volume hospitals in USA, a 38\% reduction in cardiac catheterizations of STEMI, was also noted [5]. Similarly, Tam et al. [6] reported large delays in the number of patients with STEMI seeking medical care in Hong-Kong, China, after the institution of infection control measures. Similar observations have been reported in a study of 19 public primary PCI centers in Austria, where lower rate of admitted and therefore treated patients with ACS was reported [7]. In our experience, the number of acute STEMI cases in March and April 2020 was lower compared to the preceding years.

It is hard to explain the mystery of the "missing" emergency hospital visits. It could be speculated that patients with heart symptoms may not be seeking for medical care because of the fear of infection from SARS-COV-2. In linewith the previous, infarct-related symptoms, such as chest pain or discomfort and dyspnea could have been misinterpreted as being related to an acute respiratory infection. There are many possible reasons for the observed different rates of reduction in ACS cases and performed primary PCIs during the pandemic. They may be related to local protocols of each heart center such as the promotion of thrombolysis over primary PCI during this period [8]. Additionally, they could be associated with the location of the center (i.e., COVID-19 or non-COVID-19 dedicated hospitals). Finally, they could reflect the asynchronous spread of the disease, as some countries had more time to prepare their health system response to the global threat, learning from other counties' experience. However, in our case, the percentage of individuals who admitted from the emergency department to the Cardiology ward increased during the COVID-19 epidemic period. The later could be explained because of the severity of the cases eventually visited the hospital. We are not able to provide data regarding possible delays in care delivery for STEMI cases as this registry could not retrieve door to balloon time intervals. It should be mentioned that our hospital is not dedicated in treating COVID-19 patients. Thus, triage is used for the initial evaluation of all coming patients. This includes blood pressure, temperature and oxygen saturation measurement, together with ECG, and brief medical history taking. Patients with signs and symptoms suspicious for infection undergo screening with swab-testing for SARSCOV2 and they remain isolated, in a special Unit awaiting the test results. Test-positive patients are immediately transferred to the reference hospital for COVID-19 cases. This policy includes patients with NSTEMI or unstable angina as well. In the event of a suspicious for COVID-19 STEMI case, our local protocol dictates immediate cardiac catheterization in a dedicated laboratory with all the protective measures in place. However, to date, there were no such cases. According to a position paper released by the Hellenic Cardiological Society, in line with the instructions given from Ministry of Health (https://eody.gov.gr/), all adequate precautions were taken in the catheterization lab. 
This includes proper uniform, face shields and appliance of negative environmental pressure.

Other scientists suggested that the reduction in hospital visits could be attributed to a variety of environmental and behavioral causes associated to the lockdown that governments mandated: reduction in urban air pollution because of the automobiles transportations, abstinence from a stressful job-related environment, better adherence to cardiovascular medications and less smoking because of the "staying home" policy and consequently more time for rest and be with the family.Especially for the impact of stress, the role of the imposed quarantine on the immediate psychological consequences has been evaluated during the H1N1 2009 outbreak in a providence in China, where no significant increase in stressful experiences were observed during the quarantine period [9].

The rigorous public health measures, which are undoubtedly critical for controlling the COVID-19 epidemic, may have, unintentionally, affected the health care systems. The extent to which a community outbreak of an infectious disease, like COVID-19, influence people's behavior towards health issues, is largely unknown. It is apparent that people are afraid to be exposed to the virous, as even in nonCOVID-19 hospitals, the demand for medical consultation was reduced. This is also supported by our observation that the number of people visited the ECD and then admitted to the cardiology ward, increased in April, when the epidemic curve was flattened. Nevertheless, thoughtful concerns are raised about the behavior of people, especially those with cardiac symptoms that, if ignored, may lead to irreversible health damage. Hopefully the more serious cases would have sought for appropriate medical care, irrespective of their fear, otherwise, there is a "bomb" awaiting to blow-up. However, even less serious symptoms, like those related to uncontrolled hypertension or to a new onset of atrial fibrillation - could result in major adverse events (i.e., stroke) if remain unattended [10-13].

Moreover, the effect of quarantine on the burden of CVD has to be evaluated, too, when the preventive measures will be reduced, and life will return back to normal. Specifically, it has been reported that social distancing measures, have been associated with depression and loneliness, especially among older adults [14-16]. It is known that depression and anxiety have strong adverse effects on blood pressure levels, glycemic control, compliance to medications, as well as to CVD risk [17, 18]. Furthermore, social distancing measures may promote sedentary behavior and adoption of unhealthy dietary habits, especially for people living alone [19-21].

In contrary, it could be supported that spending more time with family members, gathering and preparing homemade lunch, may promote healthier dietary habits, family coherence and spirituality. Another issue that should also be taken into account is that COVID-19 epidemic will lead to long-lasting unfavorable economic effects, not only to individuals, but also on health systems increasing future demand for healthcare services, since all postponed elective and semi-elective procedures will accumulate, blocking hospitals capacity [22, 23].

Cardiovascular disease still is the number one cause of death and disability in the western populations and with increasing rates all over the world. It has been estimated that the number of deaths due to CVD in 2016 reached 17.9 million, which accounts for the $31 \%$ of deaths globally [24, 25]. Awareness of people for heart symptoms and risk factors control should always be a priority of public health policies and practices [23]. People with CVD or who are at high cardiovascular risk due to the presence of one or more risk factors, such as hypertension, diabetes, hyperlipidaemia, need early detection and management using counselling, lifestyle changes and appropriate medication. However, if this decline in CVD related hospital visits is "true", it is something that needs to be rigorously studied, in order to learn how to keep CVD rates down.

Acknowledgements The authors would like to thank Dr C. Fragoulis, MD, Dr T. Kalos, MD Dr P. Tolis MD and Nurse in Charge H. Baka for their valuable contribution in data collection.

Funding None.

\section{Compliance with ethical standards}

Conflict of interest The author declare that there is no conflict of interest.

\section{References}

1. Li Q, Guan X, Wu P, Wang X, Zhou L, Tong Y, Ren R, Leung KSM, Lau EHY, Wong JY, Xing X, Xiang N, Wu Y, Li C, Chen Q, Li D, Liu T, Zhao J, Li M, Tu W, Chen C, Jin L, Yang R, Wang Q, Zhou S, Wang R, Liu H, Luo Y, Liu Y, Shao G, Li H, Tao Z, Yang Y, Deng Z, Liu B, Ma Z, Zhang Y, Shi G, Lam TTY, Wu JTK, Gao GF, Cowling BJ, Yang B, Leung GM, Feng Z (2020) Early transmission dynamics in Wuhan, China, of novel coronavirus-infected pneumonia. N Engl J Med 382:1199-1207

2. Petrosillo N, Viceconte G, Ergonul O, Ippolito G, Petersen E (2020) COVID-19, SARS and MERS: are they closely related? Clin Microbiol Infect 2020:S1198-S1743

3. White HD, Chew DP (2008) Acute myocardial infarction. Lancet 372(9638):570-584

4. Rodríguez-Leor O, Cid AB (2020) STEMI care during COVID19: losing sight of the forest for the trees. JACC Case Rep. https ://doi.org/10.1016/j.jaccas.2020.04.01

5. Garcia S, Albaghdadi MS, Meraj PM, Schmidt C, Garberich R, Jaffer FA, Dixon S, Rade JJ, Tannenbaum M, Chambers J, Huang PP, Henry TD (2020) Reduction in ST-segment elevation cardiac catheterization laboratory activations in the united states during COVID-19 pandemic. J Am Coll Cardiol S0735-1097(20):34913-34915

6. Tam CF, Cheung KS, Lam S, Wong A, Yung A, Sze M, Lam YM, Chan C, Tsang TC, Tsui M, Tse HF, Siu CW (2020) Impact of 
coronavirus disease 2019 (COVID-19) outbreak on ST-segmentelevation myocardial infarction care in Hong Kong, China. Circ Cardiovasc Qual Outcomes 13(4):e006631

7. Metzler B, Siostrzonek P, Binder RK, Bauer A, Reinstadler SJ (2020) Decline of acute coronary syndrome admissions in Austria since the outbreak of COVID-19: the pandemic response causes cardiac collateral damage. Eur Heart J. 1:ehaa314

8. Sörensen NA, Nikorowitsch J, Neumann JT, Rübsamen N, Goßling A, Hartikainen TS, Blankenberg S, Westermann D, Zeller T, Karakas M (2019) Predictive value of soluble urokinasetype plasminogen activator receptor for mortality in patients with suspected myocardial infarction. Clin Res Cardiol 108(12):13861393. https://doi.org/10.1007/s00392-019-01475-1

9. Wang Y, Xu B, Zhao G, Cao R, He X, Fu S (2011) Is quarantine related to immediate negative psychological consequences during the 2009 H1N1 epidemic? Gen Hosp Psychiatry 33(1):75-77

10. Miró Ò, Gil VÍ, Martín-Sánchez FJ, Jacob J, Herrero P, Alquézar A, Llauger L, Aguiló S, Martínez G, Ríos J, Domínguez-Rodríguez A, Harjola VP, Müller C, Parissis J, Peacock WF, Llorens $P$ (2018) Short-term outcomes of heart failure patients with reduced and preserved ejection fraction after acute decompensation according to the final destination after emergency department care. Clin Res Cardiol 107(8):698-710. https://doi.org/10.1007/ s00392-018-1237-z

11. Pilecky D, Vamos M, Bogyi P, Muk B, Stauder D, Racz H, Nyolczas N, Duray GZ, Zacher G, Zima E (2019) Risk of cardiac arrhythmias after electrical accident: a single-center study of 480 patients. Clin Res Cardiol 108(8):901-908. https://doi. org/10.1007/s00392-019-01420-2

12. Romero R, Gaytán JM, Aguirre A, Llorens P, Gil V, Herrero P, Jacob J, Martín-Sánchez FJ, Pérez-Durá MJ, Alquézar A, López ML, Roset À, Peacock WF, Hollander JE, Coll-Vinent B, Miró Ò (2019) The role of atrial fibrillation in the short-term outcomes of patients with acute heart failure. Clin Res Cardiol 108(6):622633. https://doi.org/10.1007/s00392-018-1389-x

13. Chouihed T, Rossignol P, Bassand A, Duarte K, Kobayashi M, Jaeger D, Sadoune S, Buessler A, Nace L, Giacomin G, Hutter T, Barbé F, Salignac S, Jay N, Zannad F, Girerd N (2019) Diagnostic and prognostic value of plasma volume status at emergency department admission in dyspneic patients: results from the PARADISE cohort. Clin Res Cardiol 108(5):563-573. https ://doi.org/10.1007/s00392-018-1388-y

14. Hansen NB, Harrison B, Fambro S, Bodnar S, Heckman TG, Sikkema KJ (2013) The structure of coping among older adults living with HIV/AIDS and depressive symptoms. J Health Psychol 18(2):198-211
15. Vasileiou K, Barnett J, Barreto M, Vines J, Atkinson M, Lawson S, Wilson M (2017) Experiences of loneliness associated with being an informal caregiver: a qualitative investigation. Front Psychol 19(8):585

16. Jawaid A (2020) Protecting older adults during social distancing. Science 368(6487): 145

17. Wei J, Hou R, Zhang X, Xu H, Xie L, Chandrasekar EK, Ying M, Goodman M (2019) The association of late-life depression with all-cause and cardiovascular mortality among communitydwelling older adults: systematic review and meta-analysis. $\mathrm{Br}$ JPsychiatry 215(2):449-455

18. Lichtman JH, Froelicher ES, Blumenthal JA, Carney RM, Doering LV, Frasure-Smith N, Freedland KE, Jaffe AS, Leifheit-Limson EC, Sheps DS, Vaccarino V, Wulsin L, American Heart Association Statistics Committee of the Council on Epidemiology, and Prevention, and the Council on Cardiovascular, and Stroke Nursing (2014) Depression as a risk factor for poor prognosis among patients with acute coronary syndrome: systematic review and recommendations: a scientific statement from the American Heart Association. Circulation 129(12):1350-1369

19. Ong AD, Uchino BN, Wethington E (2016) Loneliness and health in older adults: a mini-review and synthesis. Gerontology 62(4):443-449

20. Li G, Li H, Lu J (2020) No adequate evidence indicating hypertension as an independent risk factor for COVID-19 severity. Clin Res Cardiol. https://doi.org/10.1007/s00392-020-01653-6

21. Li B, Yang J, Zhao F, Zhi L, Wang X, Liu L, Bi Z, Zhao Y (2020) Prevalence and impact of cardiovascular metabolic diseases on COVID-19 in China. Clin Res Cardiol 109(5):531-538. https:// doi.org/10.1007/s00392-020-01626-9

22. Liu PP, Blet A, Smyth D, Li H (2020) The science underlying COVID-19: implications for the cardiovascular system. Circulation. https://doi.org/10.1161/CIRCULATIONAHA.120.047549

23. Carter P, Anderson M, Mossialos E (2020) Health system, public health, and economic implications of managing COVID-19 from a cardiovascular perspective. Eur Heart J. 22:ehaa342

24. Timmis A, Gale CP, Flather M, Maniadakis N, Vardas P (2018) Cardiovascular disease statistics from the European atlas: inequalities between high- and middle-income member countries of the ESC. Eur Heart J Qual Care Clin Outcomes 4(1):1-3

25. Thomas H, Diamond J, Vieco A, Chaudhuri S, Shinnar E, Cromer S, Perel P, Mensah GA, Narula J, Johnson CO, Roth GA, Moran AE (2018) Global atlas of cardiovascular disease 2000-2016: the path to prevention and control. Glob Heart 13(3):143-163 